AIAA-91-0579

$$
A 91-19369
$$

\title{
NUMERICAL SIMULATION OF GAS-PHASE DETONATION TRANSMISSION
}

Elaine S. Oran

Laboratory for Computational Physics and Fluid Dynamics Naval Research Laboratory, Washington, DC 20375

David A. Jones

Materials Research Laboratory

Victoria, Australia

Martin Sichel

Department of Aerospace Engineering

University of Michigan

Ann Arbor, Michigan

\section{9th Aerospace Sciences Meeting} January 7-10, 1991/Reno, Nevada 
Elaine S. Oran

Laboratory for Computational Physics

Naval Research Laboratory

Washington, D.C.

David A. Jones

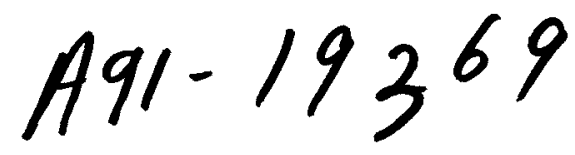

Materials Research Laboratory

Victoria, Australia

Martin Sichel

Department of Aerospace Engineering

University of Michigan

Ann Arbor, Michigan

\begin{abstract}
Time-dependent two-dimensional simulations are presented of the lateral transmission of a detonation from a stoichiometric hydrogen-oxygen mixture diluted with argon into a more dilute mixture. For the case simulated, the resulting detonation appears to die out as the leading shock becomes decoupled from the reaction front. However, it then reignites as a result of a series of shock reflections and propagates as a complex, nonsteady dynamic structure. The lowest velocity of the complex structure is always greater than the ChapmanJouguet velocity of the lean mixture and it increases in time appearing to approach the Chapman-Jouguet velocity of the stoichiometric mixture. The dynamics of the decay and reignition are described.
\end{abstract}

\section{Introduction}

The interaction between a propagating detonation and a bounding energetic medium can have a significant influence both on the detonation itself and on the way energy is transmitted into the bounding medium. When a detonation propagating through an energetic materiale first comes into contact with the bounding or secondary material, there is an initial transient phase during which the basic interaction pattern becomes established. After that, there are a number of possible types of structures that can form in the bounding material, the exact nature of which depends on the specific properties of the two materials as well as on the degree of overdrive of the incident detonation. Figure 1, a schematic of a detonation propagating through a layered material, shows the blast wave transmitted (the explosive bubble) and two of the simpler possibilities for subsequent structures: an oblique detonation with Mach reflection from the lower wall and an oblique shock with regular reflection.

In the case of gas-phase explosives, the interface between the primary detonation products and the secondary explosive acts as a high-speed gaseous wedge that can induce an oblique detonation in the secondary explosive, as shown on the far right of Figure 1. Such oblique detonations are the basis of the obliquedetonation ramjet engine that has been proposed as a supersonic combustion propulsion system [1] , and also are a fundamental component of the detonation-driven hypervelocity accelerators [2]. It is likely that reactive shock and detonation waves similar to those observed in layered detonations will arise in supersonic propulsion systems such as the supersonic combustion ramjet.

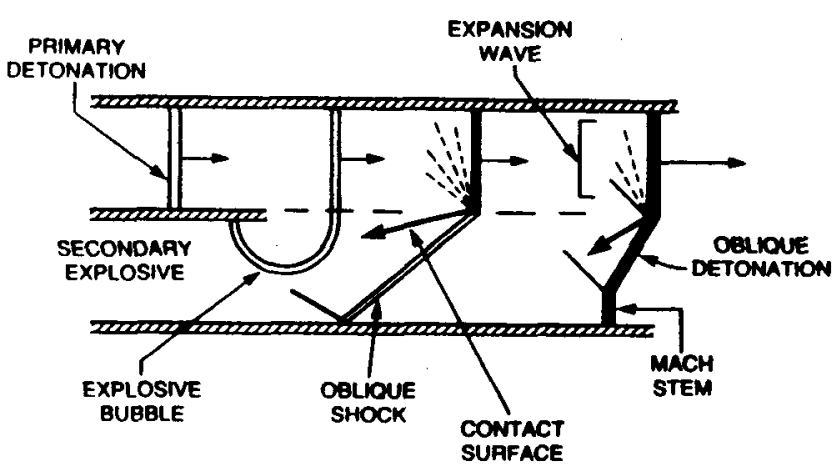

Figure 1. Typical patterns for layered detonation interaction.

For detonations in layered condensed explosives, the bounding medium generally has properties different from those of the primary explosive. An important practical question in the design and use of layered explosives is whether a suitable combination of explosives can enhance the effectiveness of the energy transmission compared to using a pure explosive with the same total energy. If the primary and bounding explosives are identical, the layered detonation pattern represents the diffraction that occurs when a detonation propagates past a step or an increase in cross-sectional area [3]. If the wall of the upper shock tube is considered as an axis of symmetry, the layered detonation also can represent the propagation of a detonation from a smaller into a larger tube and then, as indicated by [4], 
the diameter of the smaller tube must exceed a certain critical value if a detonation is to be initiated in the larger tube. This type of interaction also shows the effects of the presence of a lower reflecting wall on the critical detonation diameter. If the wall of the lower channel is considered the axis of symmetry, the layered detonation simulates an inner explosive sheathed in an outer or primary explosive.

We have now begun to compare the results of experiments and theoretical predictions [5-10] to the results of detailed time-dependent numerical simulations. The previous numerical simulations $[11,12]$ compared experimental schlieren framing photographs with comparable numerical simulations of layered detonations. These calculations modeled the layered detonations by solving the compressible two-dimensional unsteady equations for a chemically reactive flow, and then the resulting interaction patterns were compared to those observed for gaseous explosives under similar conditions. Direct quantitative comparisons were not yet possible because the simulated and experimental mixtures were not exactly the same. However, the simulations reproduced the main interaction configurations observed in the experiments. The simulation then allowed detailed exploration of the behavior of other fluid dynamic variables that could not readily be measured and thus provided additional insight into the physics of the layered detonation interaction. Thus the numerical simulations were effectively used to interpret the experimental results and to extrapolate to conditions beyond those of the experiments.

In this paper, we use time-dependent twodimensional numerical simulations to examine the detonation transmission from a dilute, stoichiometric gas to a dilute, lean gas. In particular, we describe the results of one particularly interesting simulation of a Chapman-Jouguet (CJ) detonation propagating in a tube bounded by a leaner mixture. In a previous simulation, a strong blast wave was used to initiate the interaction process [11] by depositing a fixed amount of energy in the primary explosive just upstream of the interaction region, and this is analogous to intiation using a strong blast wave. A steady interaction pattern was rapidly established in the lean, bounding explosive mixture. Here we present computations in which the initiating wave is a CJ detonation. Now when the detonation comes into contact with the lean mixture, it initially decays, but then reignites as a result of a series of shock reflections. It then propagates as an extremely complex, dynamic structure, perhaps on the brink of death, but not dying. By the time the simulation is stopped, it has accelerated to a velocity intermediate between the CJ velocity of the two mixtures and is still increasing in velocity. More details of this simulation and the model used are given in [13].

\section{MODEL AND METHOD OF SOLUTION}

Numerical simulations of gas-phase detonations are based on solutions of the compressible, timedependent, conservation equations for total mass density $\rho$, momentum $\rho \mathbf{v}$, energy $E$, and individual species number densities, assuming an ideal-gas equation of state. The effects of molecular diffusion, thermal conduction, and radiative diffusion have been omitted from these equations. The first two of these effects are generally insignificant on the time scales of interest for detonations and the last is not significant for the hydrogenoxygen systems of interest here.

In all of the calculations described here, the full set of elementary chemical reactions are not included in the model. Instead, we use the induction parameter model that reproduces the essential features of the chemical reaction and energy release process. In the earliest form of this model, three quantities are tabulated as a function of temperature, pressure, and stoichiometry: the chemical induction time, the time during which energy release actually takes place, and the amount of energy released. These quantities may be obtained by integrating the full set of elementary chemical reactions, as we have done for hydrogen-oxygen combustion in this paper, or they may be gathered from experimental data, as we have done previously for liquid nitromethane. Then a quantity called the induction parameter is defined and convected with the fluid in a Lagrangian manner. This parameter records the temperature history of a fluid element and, when the element is heated long enough, energy release is initiated. Such a model works becase it reproduces the temperature dependence and energy-release properties of the chemical reactions. It is valid as long as the computational timestep is smaller than any of the important fluid-dynamic fluctuations and for fast flows in which the convective timescales are significantly faster than those for physical diffusion. The model was first described by [14] and extended by [15-17]. Similar approaches were used by [18-21].

Here the induction parameter model is formulated in a slightly different way than first proposed. We consider two materials: the gas in the upper tube or primary explosive and the gas in the lower tube or secondary explosive, designated by subscripts 1 and 2 , respectively. Then the total density $\rho$ is the sum of the densities, $\rho=\rho_{1}+\rho_{2}$. The two gases are initially separated in the upper and lower detonation tubes but subsequently come into contact as the detonation in the upper tube encounters the end of the dividing barrier. The chemical transformations that can occur in each gas or mixture of gases proceed by a two-step process that models the full details of the interactions among the species. The first describes the chemical induction period, the time during which the reactants break up, intermediate radicals are formed. Many gases, and in 
particular hydrogen-oxygen mixtures, have well defined chemical induction times. Energy release occurs in the second step and is a time of rapid reactions and formation of stable products. In hydrogen-oxygen gases, this is the time when intermediates such as atomic hydrogen, atomic oxygen, and hydroxyl radicals peak and the product water is formed. In many gases, these two times are not so distinct or there are a number of intermediate or even endothermic stages. The reaction mechanism for hydrogen-oxygen gases, however, can be characterized appropriately by these two times [22], and in this sense the present model is similar to that used by [18]. It differs from [18], however, in the way the input is derived and therefore in the level of approximation in some regimes of temperature and pressure. Details of the present use of the model are given in [13].

The convective transport equations are solved using the nonlinear, fully compressible flux-corrected transport (FCT) algorithm [23,24], an explicit, conservative, monotone, positivity-preserving finite-volume method. The procedure for using the one-dimensional algorithm with direction and timestep splitting to produce two-dimensional or three-dimensional solutions as well as a number of related calculations performed with it are described in [24]. Then those parts of the coupled set of equations that describe the chemical reactions are solved separately and then combined with the FCT solutions for convective transport by time-step splitting methods [24].

\section{THE PHYSICAL PROBLEM}

The primary, planar detonation is propagating in a mixture with properties based on those of a dilute, stoichiometric mixture of molecular hydrogen, oxygen, and argon (2:1:7) in a detonation tube $1.6 \mathrm{~cm}$ high and $20 \mathrm{~cm}$ long. This tube rests on another tube, also $1.6 \mathrm{~cm}$ high, and is separated from it by a solid barrier that exists until the position $4.0 \mathrm{~cm}$, when the upper and lower tubes come in contact. These physical dimensions correspond to the experimental apparatus used in $[5,6]$. The properties of the secondary mixture represent a leaner mixture with correspondingly less energy release, close to the properties of a mixture with a equivalence ratio of 0.25 . The gases are all initially at 1 atm and $298 \mathrm{~K}$.

The properties of the mixtures and the detonations they support were calculated from one-dimensional computations in which a fixed amount of energy was deposited near the origin in the form of excess temperature and pressure. The result of such computations is an overdriven detonation which, in time, decays to CJ conditions. After running such a computation for 20,000 time steps, the detonation velocity was essentially constant. The final one-dimensional profiles of density, momentum, energy, pressure, and temperature around the detonation front were used to start the planar detonation in the two-dimensional computations. The CJ velocities of the primary and secondary mixtures were $1560 \mathrm{~m} / \mathrm{s}$ and $1000 \mathrm{~m} / \mathrm{s}$, respectively. The detonation cell sizes for these mixtures are $0.1 \mathrm{~cm}$ and $0.5 \mathrm{~cm}$, respectively, as estimated from data from Strehlow [25] for three-dimensional detonations. (For two-dimensional, planar detonations, we would expect the cell sizes to be somewhat different.)

Parameters in the model are chosen so that the detonation has the CJ velocity corresponding to the mixture modeled. The energy release for the primary and secondary mixtures was $0.180 \mathrm{kcal} / \mathrm{g}$ and $0.070 \mathrm{kcal} / \mathrm{g}$, respectively, and the molecular weights were 31.6 and $60.0 \mathrm{amu}$. Values for the reaction parameters $A_{r}, E_{r}, A_{\tau}$, and $E_{\tau}$ are $2.4 \times 10^{8} \mathrm{~s}^{-1}, 3.0 \times 10^{4}$ kcal, $5.6840 \times 10^{-8} \mathrm{~s}^{-1}$, and $1.5031 \times 10^{4} \mathrm{kcal}$, respectively, for both mixtures. In addition, the value of $\gamma$ is held constant in the calculation: it is not allowed to be a function of composition or temperature. This is certainly an unphysical assumption. Here, however, this is part of the simplified parametric model that ensures that the values of certain global physical quantities, such as the detonation velocity, have the correct physical value. Selecting and fitting these parameters must be viewed in the same way and are at the same level of approximation as the induction parameter model.

The simulations were carried out on an evenly spaced rectangular grid with $\Delta x=\Delta y=0.04 \mathrm{~cm}$. The computational domain had 500 computatonal cells in the $x$-direction and 80 cells in the $y$-direction. An average numerical time step, calculated by taking one quarter of the value required by the Courant condition, is approximately $5 \times 10^{-8} \mathrm{~s}$.

A number of resolution and other detailed numerical tests were done using these model mixtures and are reported by [26]. The essential results are that for the grid size chosen, the profiles of the one-dimensional CJ detonation do not vary with change in $\Delta x$. Thus for some purposes, and these will be discussed further in the Discussion section, the detonation computations presented below are adequately resolved.

\section{INTERACTION OF DETONATION LAYERS}

Figure 2 shows a series of pressure contours for the problem described above and provides a summary of the physical problem. By timestep 200, the detonation reaches a location where there is no longer a barrier separating it from the mixture in the bottom tube. It then expands as a bubble into the lean mixture below as it also continues to propagate in the upper stoichiometric mixture. By step 800 , the system has evolved so that the detonation on the top is connected to a very oblique leading shock on the bottom, and the bubble resulting from the the transmission process has reflected from the wall. Then the entire system continues to weaken, except for the complex shock structure formed 
when the bubble reflects from the lower wall. This reflected shock structure steepens and some time after step 2000, the structure becomes very intense and appears to ignite near the Mach stem formed on the bottom. By steps 2400 or 2600 , there are two very strong waves, one on top and one on the bottom, and these continue to grow to form what appears to be a reestablished propagating detonation. Figure 3 , a history of the mean velocity of the overall structure as determined by the velocity of the leading shock wave, shows that after the detonation reaches the barrier, its velocity decreases with time until a point where it starts to increase again.

In the material that follows, we examine this extinction and reignition problem in more detail by describing. 1) the initial detonation transmission and expanding bubble, 2) the detonation decay and reignition in the upper, primary material, 3) the shock and reignition in the secondary material, and then 4) the overall interaction process.
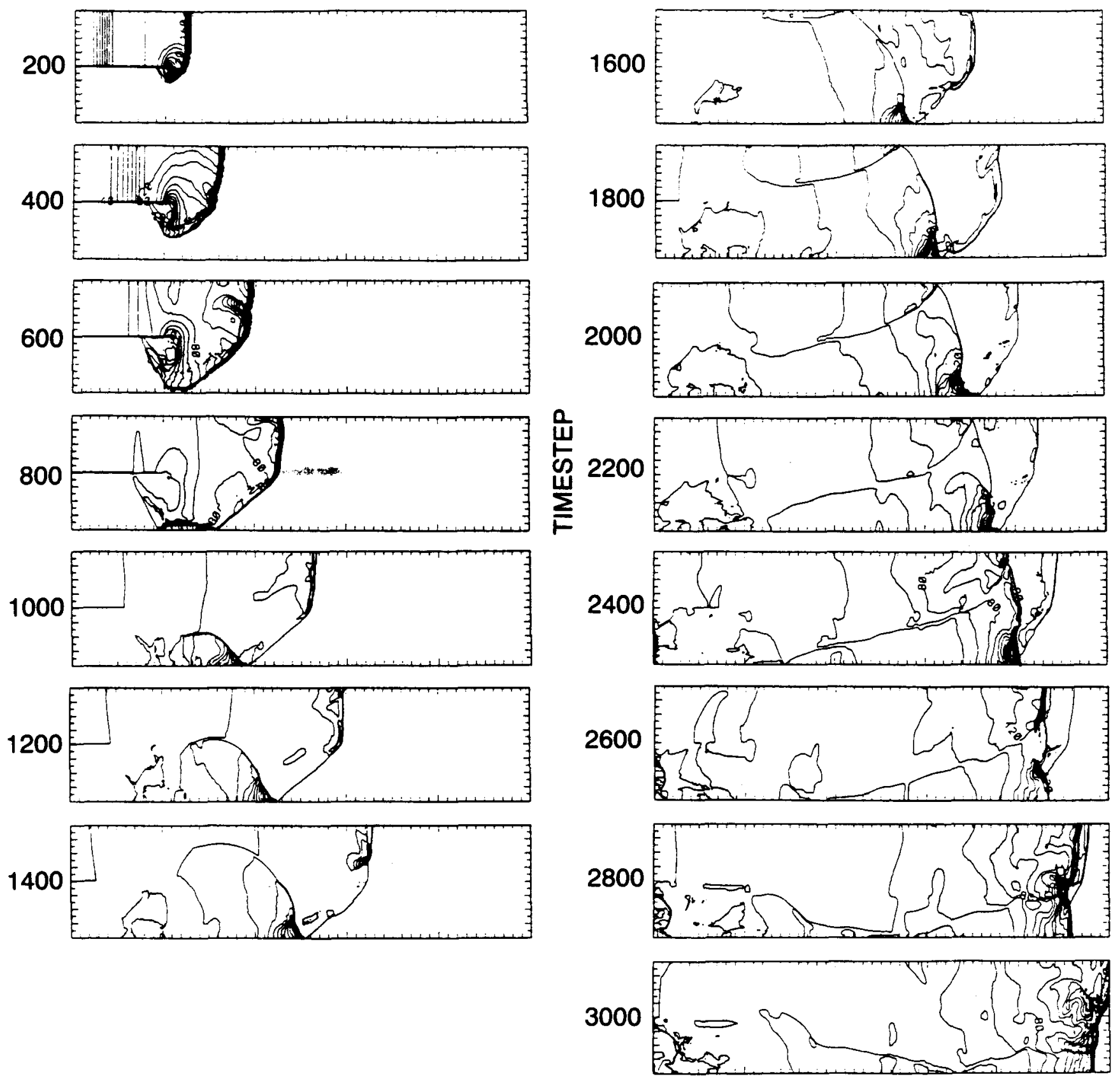

Figure 2. Pressure contours at selected timesteps for a series of contours for the transmitted layered detonation. The vertical height is $3.2 \mathrm{~cm}$ and the horizontal expanse is $20 \mathrm{~cm}$. One timestep is approximately $5.0 \times 10^{-8}$ seconds. 


\section{Detonation Transmission}

As the detonation expands into the lower tube, the expansion causes the leading shock to decouple from the reaction front and a blast wave moves down into the lean material. Below we refer to this blast wave as a bubble because it consists of less dense, but highertemperature material than the surrounding medium into which it is propagating. Where the flows turns around the barrier, a complex vortical structure forms. Some time before step 700, the bubble shock reflects from the bottom wall and then moves upward, so that at some time before step 1000 , as seen in Figure 2, it merges with the contact surface above it. This reflected shock further heats and compresses the unreacted material behind it, perhaps even igniting it. Figure 2 also shows a transverse wave structure at the detonation front in the top half of the tube. This structure initially appears at the contact surface between the two materials and it seems to have been generated by the disturbance whose orgin is the interface between the two materials. In time, the structure moves upward and at step 700 , the tail of the wave meets the top bounding wall.

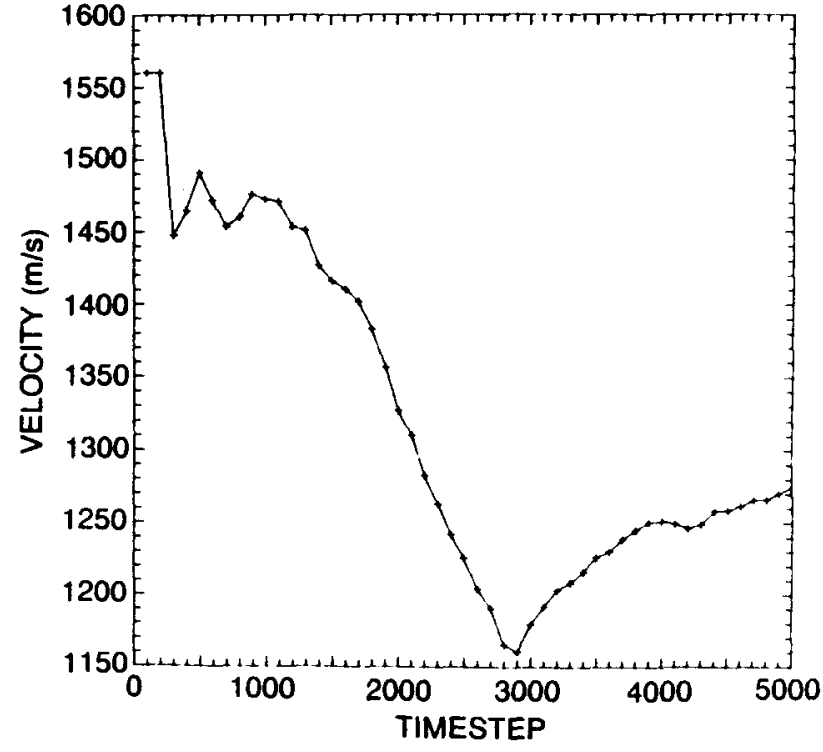

Figure 3. Velocity of the shock-detonation complex.

TIMESTEP 1200

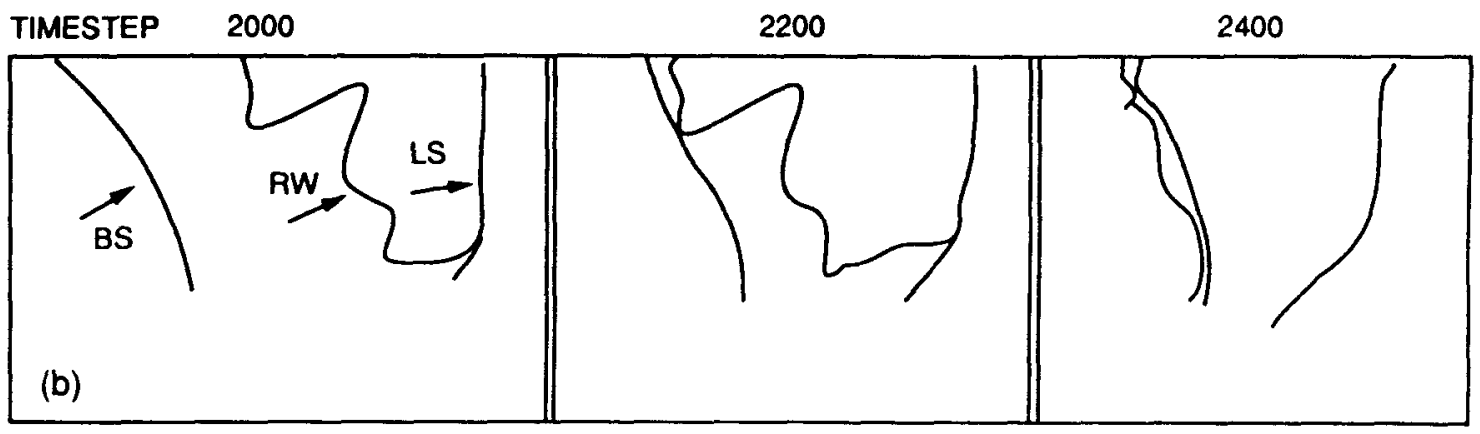

Figure 4. Tracings taken from pressure, temperature, and density contours in the upper tube showing a) the leading shock (LS) and reaction wave (RW) and b) the leading shock, reaction wave, and bubble shock (BS).

\section{Decay of the Primary Detonation}

The originally planar detonation wave propagating in the top channel is weakened by the expansion process occurring below it. For some time, this upper wave structure is characterized by a closely coupled leading shock and a reaction front, at least through step 


\section{Decay of the Primary Detonation}

The originally planar detonation wave propagating in the top channel is weakened by the expansion process occurring below it. For some time, this upper wave structure is characterized by a closely coupled leading shock and a reaction front, at least through step 1400. At step 1200, the coupling between the shock and reaction front is still fairly close so that this upper wave structure might still be called a detonation. Both the temperature and density contours show the contact discontinuity between the products of the primary mixture and the unburned secondary mixture behind the oblique shock wave. However, between steps 1600 and 1800 , the shock and reaction front become more and more decoupled to the point that the upper structure can no longer be called a detonation and, as shown in
Figure 3, the primary front continues to decelerate.

The Reflected Bubble Shock

Upstream of the leading shock structure, there is a secondary shock that has developed from the reflection of the bubble off the lower wall. This reflected bubble shock is first evident at step 800 . As this shock moves upward in the chamber, it moves first into unreacted lean gas and then into the fully reacted gas in which the primary detonation was propagating. As it moves upwards, it accelerates and by step 1600, it hits the top wall and produces another reflected shock moving downwards and upstream into fully reacted material and away from the propagating fronts. This second reflected shock subsequently sweeps down in the tube behind the bulk of the structure and finally reflects from the bottom wall near step 2400 .
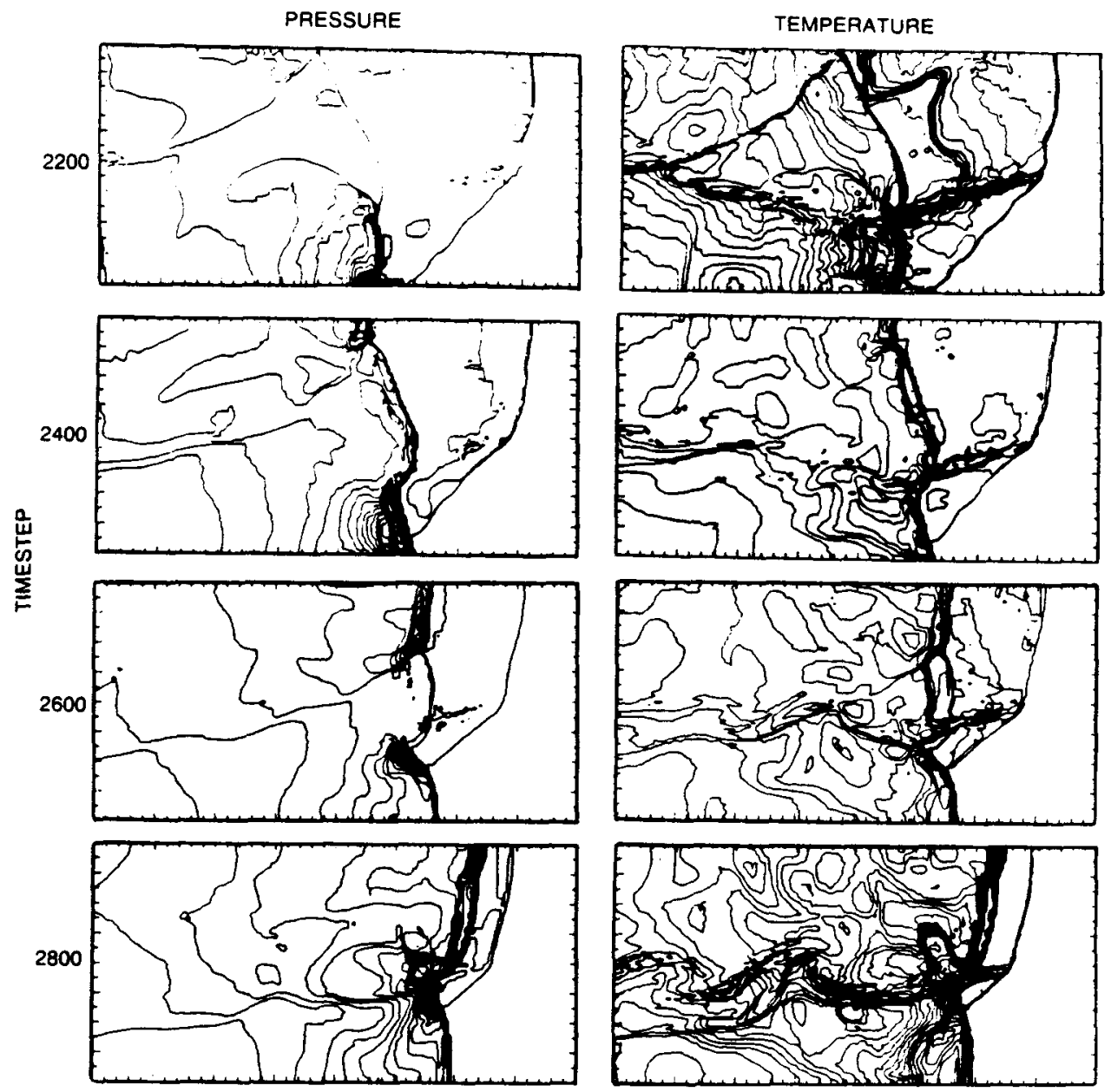

Figure 5. Pressure and temperature contours for steps 2200, 2400, 2600, and 2800 showing reignition in the primary and secondary mixtures.

\section{Reignition of the Detonation Wave}

Figure 4 consists of a series of traces of selected parts of the shock and reaction wave in the upper tube. This figure contains a composite of information taken from pressure, density, temperature, and reaction-variable contours. Figure $4 \mathrm{a}$ is a sequence showing just the leading shock and the reaction wave. By step 2000, the reaction front and the leading shock are still sepa- 


\section{Reignition of the Detonation Wave}

Figure 4 consists of a series of traces of selected parts of the shock and reaction wave in the upper tube. This figure contains a composite of information taken from pressure, density, temperature, and reaction-variable contours. Figure $4 \mathrm{a}$ is a sequence showing just the leading shock and the reaction wave. By step 2000, the reaction front and the leading shock are still separating. Figure $4 \mathrm{~b}$ repeats step 2000 , but now includes the upstream shock that resulted from the bubble reflection. In time, this shock steepens and eventually meets the decaying reaction wave in step 2200 . By step 2400 , the bubble shock and the reaction wave are now a closely coupled complex, looking somewhat like a detonation, and overtaking the leading shock. This sudden merging of the flame and shock wave initiates the process which, by step 2900 , leads to reacceleration of the leading shock front.

The reignition that occurs in the upper tube is a key phenomenon that occurs when a shock wave overtakes a reaction wave, flame, or deflagration. After the shock overtakes the reaction wave, the complex that is formed (the two close contours at step 2400 in Figure $4 \mathrm{~b}$ ) accelerates with respect to the leading shock. This new complex reaction wave is moving at approximately $1600 \mathrm{~m} / \mathrm{s}$, which is substantially above the $1200 \mathrm{~m} / \mathrm{s}$ of the leading shock, and supersonic with respect to the material behind the leading shock. The temperature behind the leading shock is $1200 \mathrm{~K}$, and the speed of sound in this material is about $700 \mathrm{~m} / \mathrm{s}$, so that this complex is a strong, high-speed reaction wave moving through this material. The reignition process seems to be associated with the strengthening of the bubble shock.
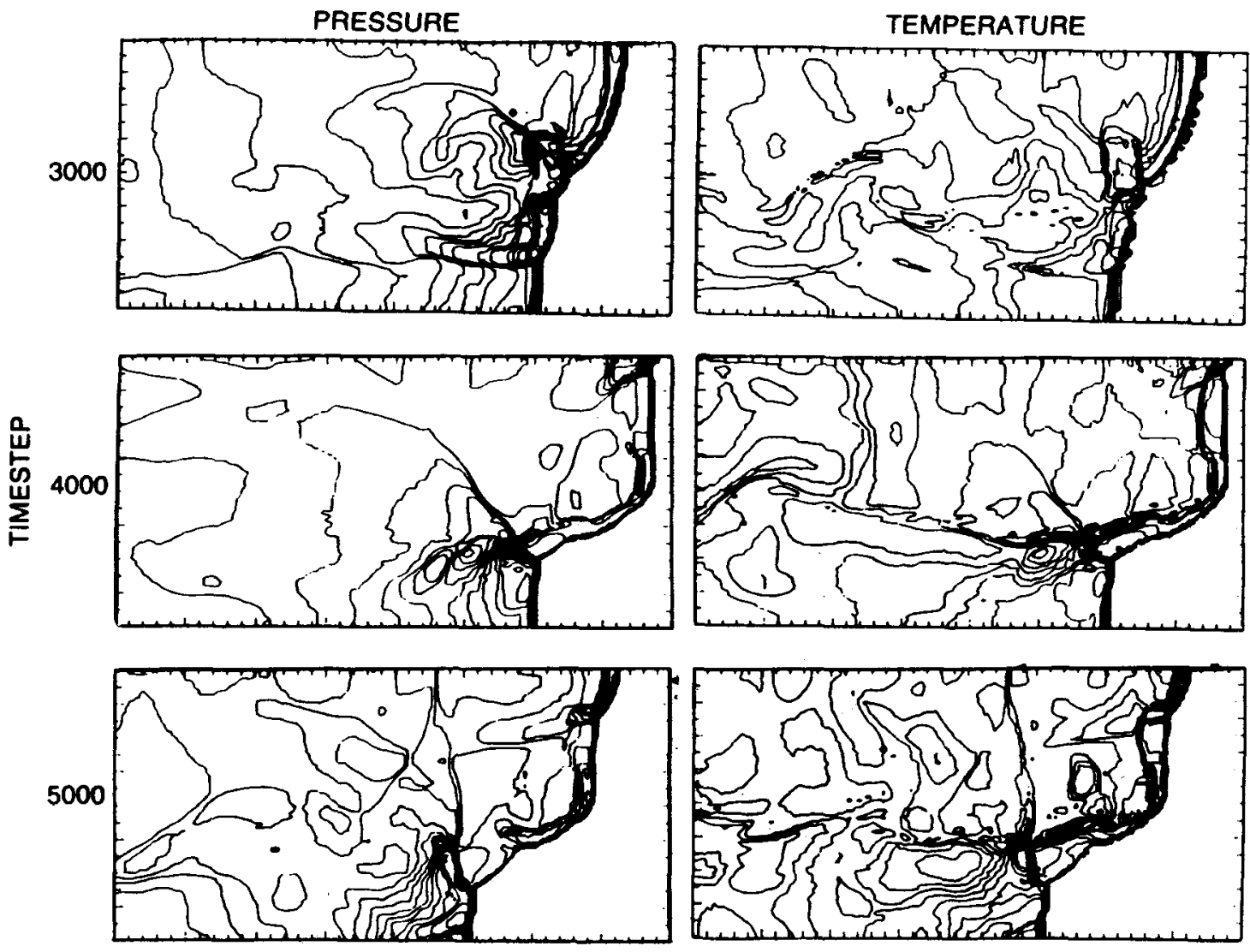

Figure 6. Pressure and temperature contours for late-time steps 3000, 4000, and 5000.

Ignition of a detonation structure in the lower tube occurs some time between steps 2000 and 2200 near the lower reflecting wall. Figure 5 shows a composite and enlargement of contours for steps 2200 through 2800 . The reaction process occurring on the bottom at step 2200 is similar to a mode of ignition observed in previous simulations and described in detail by [11] and [12]. Here it intensifies the bubble shock, which meets the upper reaction wave and becomes a detonation structure in the upper tube, evident in step 2400. Step 2600 shows complex combined shock and detonation structures propagating toward each other from the upper and lower reflecting surfaces. The pressure contours show closely coupled detonation structures on 
both the top and bottom, connected by a curved shock in the center. The original leading shock still leads the detonations on top and bottom. By step 2800, the upper detonation is even closer to the leading shock, and by step 3000, as shown in Figure 10, it has reached the leading shock and combined with it to form one structure. Until this time, the velocity of the combined detonation structure has been constantly decaying to below the CJ value of the upper mixture, but it has never decayed to below the CJ velocity of the lower mixture. At around step 2900, as seen in the velocityhistory profile in Figure 3, the velocity of the combined structure suddenly begins to increase.

\section{The Final (?) State}

In computations of the type reported here and in experiments, there is always the question whether the detonating system has reached a final steady state mode of propagation. The present calculations have been extended to 5000 time steps. By this time reignition has occurred separately in both the top and bottom mixtures and the resultant detonations have merged to form the configuration shown in Figure 6. The system has evolved into an approximately normal detonation in the primary mixture connnected by an oblique shock to a detonation in the lower, secondary mixture. This configuration is similar to those observed experimentally [6] when the primary $\mathrm{H}_{2}-\mathrm{O}_{2}$ mixture is stoichiometric and the secondary mixture is lean, but here, too, it is not entirely clear whether the interaction has reached a final steady state at the end of the observation period. Figure 3 shows that by the time the computation was terminated at step 5000 , the velocity of the complex had not reached its final value, but was still increasing. An extrapolation from this figure indicates that the velocity might reach the CJ velocity of $1560 \mathrm{~m} / \mathrm{s}$ in the primary mixture by step 15,000 .

\section{DISCUSSION}

We have presented a detailed description of one particularly interesting computation of the lateral transmission of a CJ detonation in a stoichiometric hydrogenoxygen mixture into a lean hydrogen-oxygen mixture, both diluted with argon. This computation was one of a series done to help interpret the results of laboratory experiments, but in itself has provided some insight into mechanisms of detonation propagation and decay. The situation presented here is dynamic and complex and cannot be described by classical steady-state theories based on shock and detonation polars, although the results of such analysis do provide some guide to the final configuration [7]. Thus when the secondary wave is sufficiently overdriven by the primary wave, the polar theory predicts that the transmitted wave will be either an oblique shock or, if conditions are favorable to initiation, an oblique detonation. As already indicated, this simple theory provides no indication as to which of these final configuration will arise; this depends on unsteady processes of the type treated here.

A planar detonation propagates at the CJ velocity in a medium bounded by perfectly reflecting walls. If the computational resolution were high enough and the system were perturbed, we would see the planar front change to one with dynamically changing shock structure $[14,15,27]$. In fact, a real detonation in this mixture has a complex structure at the front consisting of interacting incident shocks, Mach stems, and transverse waves that produce the complex pattern of triple points that, when regular enough, lead to what are called detonation cells.

However, because the numerical algorithm is conservative, with the choice of model parameters the detonation propagates at the correct velocity, and structures on scales large compared to the cell size are expected to be fairly accurate. This is substantiated by the similarity noted by [11] among computational and laboratory experimental results, and we generally expect this to be true as long as the detonation cells are small enough compared to the larger-scale flow structures of interest here. We therefore assume that the multidimensional calculation on the scale of the phenomena observed is converged enough so that further refining the computational grid would only show more small-scale structure, but not change the basic properties of the large-scale structure we see. This last observation has been verified to some extent in a series of extremely resolved computations [28] and by the agreement between the simulated and observed detonation diffraction patterns at a step.

Eventually the lower bounding wall of the primary tube terminates and the one-dimensional detonation expands into a leaner mixture. As it expands, the reaction front and shock front decouple so that a shock is expanding into the lower mixture and moving generally towards the lower bounding wall. This bubble shock eventually hits the lower wall and is reflected, so that it then moves upward and interacts with the contact surface above it that separates reacted from nonreacted gas. As the bubble shock moves upwards, its vertical velocity increases because of a change in the properties of the gas it moves through (hot, lowerdensity) and it might also gain some impetus from any ignition of unburned lean material that occurs behind it.

After the initial detonation transmission, the detonation continues to propagate on the top channel, continuously decaying, as indicated by the velocity history in Figure 3 and the increase in the size of the induction zone behind the leading shock. Eventually, sudden explosive reignition occurs in the Mach stem of the reflected bubble shock. Experiments have been reported [27] which studied the propagation of quasi- 
detonations, which are detonations that propagate in very rough tubes at speeds substantially below the CJ velocity. In a series of experiments showing the propagation of a detonation over an obstacle and the diffraction of a detonation from a corner, they note the importance of shock reflections from the wall in the overall reinitiation process. If they delayed the reflections by changing the spacing of obstacles or walls, the transition to the quasi-detonation state is delayed. These experiments also show that if the Mach stem formed on shock reflection is strong enough, the detonation can reignite.

The exact physical mechanism by which this reignition occurs is not clear and two different mechanisms are possible here: the detonation can reignite by autoignition as a consequence of adiabatic shock heating or vortex mixing in the shear layer near the wall behind the Mach stem. It is clear from many computations that the wave structure behind the Mach stem is complex and there are regions where shocks intersect and the temperature reaches ignition temperature. There are also shear layers entraining and mixing material that has chemically reacted in varying amounts. However, in the present simulations, it appears that the high temperatures themselves in the region of the Mach stem would be sufficient to ignite the mixture, even though it was previously primed by the mixing process. This ignition near the Mach stem coincides with an acceleration and straightening of the bubble shock so that it interacts with the reaction front in the upper wave structure. The interaction of the bubble shock and reaction front accelerates the reaction front so that a detonation is formed in the top layer and this detonation moves and combines eventually with the leading shock to form one structure.

There are several points on which it is interesting to speculate. First, if the tube were wider, would the detonation reignite? Another way of asking this question is to ask, how important is the bounding bottom wall in the reignition process? The process described above could not happen without the presence of the reflected bubble shock, which results in both the lower Mach stem and the upper shock-flame interaction. Even when there are no obstacles to cause reflection when a material is heated long enough, ignition occurs "spontaneously." Thus ignition might seem to occur spontaneously or perhaps the detonation might never die if either the bounding medium is more energetic or if the primary detonation is overdriven. This effect provides an alternate reignition mechanism to what we see in the situation simulated above.

In an earlier paper [11], we described a simulation with parameters almost the same as the one described above, the difference being that the primary detonation was overdriven so that at the point where the two mixtures meet, the velocity of the detonation was $54 \%$ over CJ. In this case, the transmitted bubble shock is stronger and the primary detonation did not decay as quickly in the upper tube and reestablished itself without the help of the bubble shock interacting with a decaying reaction wave. The leaner mixture in the lower tube is still ignited behind a Mach stem generated by the reflecting bubble shock. The final state is a detonation in the top and bottom tubes connected by an oblique shock, a picture similar to that observed by step 5000 in the present configuration as shown in Figure 10. If the detonation had been overdriven even further, it is possible that the bubble shock could have directly ignited the secondary mixture.

If the detonation does not completely die out, which is the case in the simulation shown above, there are several possible outcomes. First, a combined detonation structure might propagate at some average of the detonation velocities of the upper and lower structure, and this is what we see. However, other extremes are possible. For example, it is possible to imagine an even more dynamic scenario where the detonation in the upper tube continually or cyclically dies and reignites. This phenomenon very much resembles the galloping detonations discussed [30]. The detonation could completely die out immediately. The range of possibilities here no doubt depends on the material properties as well as the width of the tube. Suppose the bottom tube were wider, then the velocity of the total structure might go below its CJ velocity before reflection of the bubble shock, and then it is possible that reginition might not occur.

Further confirmation that the simulation used here can reproduce the main features of the layered explosive interaction is provided by the comparison presented in [31] of the framing Schlieren photographs of the diffraction at a step of a stoichiometric $\mathrm{H}_{2}-\mathrm{O}_{2}$ detonation at a pressure of 120 torr [29] and the results of the simulation of a layered detonation with both primary and secondary mixtures the same as the primary mixture above [11]. A comparison of Fig. 6 from Teodorczyk with Figure 10 from [11] showed that the main features of the diffraction were produced by the simulation. The two configurations are somewhat different: one case is diffraction at a step and the other is an interaction between two layers. The two explosive mixtures being compared also are different, but the low pressure in the experiments tends to increase induction and reaction distances in the same way as the Argon dilution of the mixture considered in the simulation. In some sense the simulation and experiment are thus equivalent, as is supported by the fact that the simulation reproduces the main features of the observed interaction.

A typical computation required approximately $25 \mu$ s/timestep/computational cell on a Cray X$\mathrm{MP} / 14$. As with many large-scale multidimensional 
calculations, we have reached the point where the computational time is considerably less than the analysis time of the output and time taken to generate the diagnostics. This is certainly true for complex twodimensional calculations and would be even more true for three-dimensional calculations, where the graphical display capabilities are even more rudimentary.

\section{Acknowledgments}

The computations were performed on a Cray X-MP/14 with funding provided by the Materials Research Laboratory. This work was sponsored in part by the Naval Research Laboratory through the Office of Naval Research and in part by U.S. Army Research Office under grant No. DAAL03-87-K-0019.

\section{References}

1. Sheng, Y., and Sislian, J.P., 1985, A Model of a Hypersonic Two-Dimensional Oblique Detonation Ram Jet, University of Toronto Institute of Aerospace Studies, Tech. Note 257.

2. Hertzberg, A., Bruckner, A.P., Bogdonoff, D.W., 1988, Ram Accelerator: A New Chemical Method for Accelerating Particles to Ultrahigh Velocities, AIAA J. 26, 195-203.

3. Bartlmä, F., and Schröder, K., 1986, The Diffraction of a Plane Detonation Wave at a Convex Corner, Combustion and Flame 66, 237-248.

4. Matsui, H., and Lee, J.H., 1979, On the Measure of the Relative Detonation Hazards of Gaseous FuelOxygen and Air Mixtures, Proceedings of the 17th Symposium (International) on Combustion), The Combustion Institute, Pittsburgh, pp. 1269-1280.

5. Liu, J.C., Liou, J.J., Sichel, M., Kauffman,C.W., and Nicholls, 1987, J.A., Diffraction and Transmission of a Detonation into a Bounding Explosive Layer, Proceedings of the 21st Symposium (International) on Combustion, The Combustion Institute, Pittsburgh, pp. 1659-1668.

6. Liu, J.C., Sichel, M., and Kaufman, C.W., 1988, The Lateral Interaction of Detonating and Detonable Gaseous Mixtures, Progress in Astronautics and Aeronautics, Vol. 114, pp. 264-283.

7. Liou, J.J., 1986, Analysis of the Wave Interaction Between a Propagating Detonation and a Bounding Explosive Layer, Ph.D. Thesis, Department of Aerospace Engineering, The University of Michigan, Ann Arbor, Michigan, MI.

8. Fan, B.C., Sichel, M., and Kauffman, C.W., 1988, Analysis of Oblique Shock-Detonation Wave Interactions in the Supersonic Flow of a Combustible Medium, AIAA Paper AIAA-88-0441.

9. Dabora, E.K., Nicholls, J.A., and Morrison, R.B., 1965, The Influence of a Compressible Boundary on the Propagation of Gaseous Detonations, Proceedings of the Tenth Symposium (International) on Combustion, p. 817.

10. Dabora, E.K., Desbordes, D., Guerraud, C., and Wagner, H.Gg., 1990, Oblique Detonation at High Velocities, to appear in Proceedings of the 12th International Colloquium on the Dynamics of Explosions and Reactive Systems, AIAA Progress Series.

11. Jones, D.A., Sichel, M., Guirguis, R., and Oran, E.S., 1990, Numerical Simulation of Layered Detonations, to appear in Progress in Aeronautics and Astronautics, AIAA, Washington.

12. Sichel, M., Jones, D.A., Oran, E.S., and Guirguis, R., 1990, Detonation Transmission in Layered Explosives, to appear, Proceedings of the 23rd Symposium (International) on Combustion.

13. E.S. Oran, D.A. Jones, and $M$. Sichel, The Anatomy of a Marginal Detonation, submitted to Proc. Roy. Soc., 1990.

14. Oran, E., Boris, J.P., Young, T.R., and Picone, J.M., 1981, Numerical Simulations of Detonations in Hydrogen-Air and Methane-Air Mixtures, Proceedings of the 18th Symposium (International) on Combustion, The Combustion Institute, Pittsburgh, PA, pp. 1641-1649.

15. Kailasanath, K., Oran, E.S., and Boris, J.P., 1985, Determination of Detonation Cell Size and the Role of Transverse Waves in Two-Dimensional Detonations, Combustion and Flame, Vol. 61, pp. 199-209.

16. Guirguis, R., Oran, E.S., and Kailasanath, K., 1986, Numerical Simulations of the Cellular Structure of Detonations in Liquid Nitromethane Regularity of the Cell Structure, Combustion and Flame, Vol. 65, pp. 339-366.

17. Guirguis, R., Oran, E.S., and Kailasanath, K., 1987, The Effect of Energy Release on the Regularity of Detonation Cells in Liquid Nitromethane, Proceedings of the 21st Symposium (International) on Combustion, The Combustion Institute, Pittsburgh, pp. 1659-1668,

18. Korobeinikov, V.P. , Levin, V.A., Markov, V.V., and Chernyi, G.G., 1972, Astronautica Acta , 17, 529.

19. Taki, S. and Fujiwara, T., 1981, Numerical Simulation of Triple Shock Behavior of Gaseous Detonation, Eighteenth Symposium (International) on Combustion, pp. 1671-1681, The Combustion Institute, Pittsburgh, PA.

20. Kaplan, C., and Oran, E.S., 1990, A Numerical Study of Spontaneous Propane Ignition in a Partially Confined Volume, submitted to the Third International Symposium on Fire Safety Science, Edinburgh, Scotland.

21. Gubin, S.A., and Sichel, M., 1977, Calculation 
of the Detonation Velocity of a Mixture of Liquid Fuel droplets and a Gaseous Oxidizer, Comb. Sci. Tech., 17, 109-117.

22. Burks, T.L. and Oran, E.S., 1981, A Computational Study of the Chemical Kinetics of Hydrogen Combustion, NRL Memorandum Report 4446, Naval Research Laboratory.

23. Boris, J.P., and Book, D.L., 1976, Solution of the Continuity Equation by the Method of FluxCorrected Transport, Methods in Computational Physics, Vol. 16, pp. 85-129.

24. Oran, E.S., and Boris, J.P., 1987, Numerical Simulation of Reactive Flow, Elsevier, New York.

25. Strehlow, R.A., 1984, Combustion Fundamentals, p. 307, McGraw Hill, New York.

26. Jones, D.A., Oran, E.S., Guirguis, R., 1990, A One-Dimensional Flux-Corrected Transport Code for Detonation Calculations, to appear as an MRL Research Report, Materials Research Laboratory, Victoria, Australia, and also as an NRL Memoran- dum Report, Naval Research Laboratory, Washington, DC.

27. Oran, E.S., Kailasanath, K., and Guirguis, R.H., 1988, Numerical Simulations of the Development and Structure of Detonations, Progress in Astronautics and Aeronautics, 114, 155-169.

28. Kailasanath, K., Ganguly, K., and Oran, E.S., 1990, Detailed Structure of Two-Dimensional Detonations, K. Kailasanath and E.S. Oran, in preparation for submission to Combustion and Flame.

29. Teodorczyk, A., Lee, J.H.S., and Knystautas, R., 1989, Twenty-Second Symposium (International) on Combustion, p. 1723-1731, The Combustion Institute, Pittsburgh, PA.

30. Fickett, W., and Davis, C.W., 1979, Detonation, University of California Press, Berkeley

31. Sichel, M., 1990, Transition to Detonation - Role of Explosion within and Explosion, Proceedings of the NASA Combustion Workshop, to be published by Springer, New York. 